\title{
Environmental taxonomy of power scavenging techniques for autonomous self powered wireless sensors
}

\begin{abstract}
Power is a critical issue in wireless sensor node, because in most of the applications it is difficult or in some cases impossible to replace or replenish the battery. This research surveys, summarizes and categorize the possible solutions to harvest required power of wireless sensor node from the working environment. Sensors are divided in different categories according to their application and working environment and possible solutions for harvesting energy in each category discussed. Furthermore with applying hybrid techniques sensor node will be able to supply its own power using environmental phenomenon and whatever it senses or have access to.
\end{abstract}

Keyword: Autonomous systems; Green technologies; Power scavenging; Self powered; Sensor; Wireless sensor networks 